\title{
DISTRIBUCIÓN DE FLEBOTOMINOS (DIPTERA: PSYCHODIDAE) EN QUINTANA ROO, MÉXICO
}

\author{
Emigdio May-UC, ${ }^{1}$ Héctor A. Hernández-Arana ${ }^{1}$ y Eduardo A. \\ REBOLLAR-TÉLLEZ ${ }^{2}$ \\ ${ }^{1}$ El Colegio de la Frontera Sur unidad Chetumal, Av. Centenario km 5.5, C.P. 77014, Chetumal, \\ Quintana Roo, México.<emagyc1@yahoo.com.mx>, <hhernand@ecosur.mx> \\ ${ }^{2}$ Laboratorio de Entomología Médica, Facultad de Ciencias Biológicas. Universidad Autónoma de \\ Nuevo León, Monterrey, Nuevo León, México. <eduardo.rebollartl@uanl.edu.mx>
}

May-Uc, E., H. A. Hernández-Arana \& E. A. Rebollar-Téllez. 2011. Distribución de flebotominos (Diptera: Psychodidae) en Quintana Roo, México. Acta Zoológica Mexicana (n. s.), 27(2): 273-289.

RESUMEN. Se llevó a cabo un estudio faunístico en el estado de Quintana Roo para conocer la distribución, riqueza y abundancia de los flebotominos. Se observó una gran variabilidad del promedio de especies y abundancia dentro y entre tres diferentes zonas (sur, centro y norte) en el estado. La mayor abundancia y riqueza se registró en la zona norte, en la zona centro y sur se capturaron igual número de especies, y la zona centro tuvo la menor abundancia. En cada zona fueron diferentes las especies dominantes, aunque, Lutzomyia shannoni y Lutzomyia cruciata se registraron dentro de las más dominantes distribuidas en las tres zonas. Entre las especies dominantes que se presentan en el estado se encuentran aquellas que son incriminadas como vectores de leishmaniasis en la Península de Yucatán. Los factores ambientales que se presentan en el estado son reflejados en las abundancias encontradas en las diferentes zonas de Quintana Roo.

Palabras clave: Diptera, Psychodidae, Phlebotominae, Lutzomyia, Brumptomyia, distribución, Quintana Roo, leishmaniasis.

May-Uc, E., H. A. Hernández-Arana \& E. A. Rebollar-Téllez. 2011. Distribution of phlebotomine sandflies (Diptera: Psychodidae) in Quintana Roo, Mexico. Acta Zoológica Mexicana (n. s.), 27(2): 273-289.

ABSTRACT. A faunistic study was carried out to gain insight into the distribution, species richness and abundance of sandflies in Quintana Roo, Mexico. There was a great intra- and interregional variability in mean species richness and abundance in Quintana Roo. The northern region appeared to have the highest species richness and abundance, followed by the central and southern regions, which exhibited an equal richness between them. Also, the central region showed the lowest sandfly abundance. Each region showed a rather distinctive dominant species composition, although two species, Lutzomyia shannoni and Lutzomyia cruciata, were among the most abundant in all three regions. In fact, some of the most abundant speciesare suspected vectors of leishmaniasis at the Yucatan Peninsula. Environmental factors

Recibido: 15/12/2009; aceptado: 12/11/2010. 
prevailing in the state are thought to be the main factors mediating the differences in sandfly abundances in the different regions of Quintana Roo.

Key words: Diptera, Psychodidae, Phlebotominae, Lutzomyia, Brumptomyia, distribution, Quintana Roo, leishmaniasis.

\section{INTRODUCCIÓN}

Los flebotominos (Diptera: Psychodidae: Phlebotominae) son dípteros dentro de los cuales existen especies capaces de transmitir patógenos cuando se alimentan de sus hospederos. En el mundo se conocen poco más de 700 especies y en México se han registrado hasta ahora un total aproximado de 40 especies de flebotominos (Young \& Duncan 1994; Ibáñez-Bernal 2000). Los estados inmaduros se desarrollan en los suelos ricos en materia orgánica (Feliciangeli 2004). Los adultos son nocturnos y crepusculares, durante el día se encuentran en ambientes húmedos y oscuros, en cavidades de árboles, rocas o cuevas. Ambos sexos se alimentan de carbohidratos de plantas, las hembras por serprincipalmente anautógenas, necesitan alimentarse de la sangre de vertebrados, principalmente mamíferos (Gibson \& Torr 1999).

Se ha detectado que los flebotominos son vectores de diversos virus, rickettsias y protozoarios causantes de enfermedades. La leishmaniasis son un tipo de enfermedades causadas por un grupo de protozoarios del género Leishmania Ross, que afectan a las personas en varios países del mundo. El principal vector de la leishmaniasis en América son los flebotominos del género Lutzomyia spp. (Organización Mundial de la Salud [OMS] 1990).

La Península de Yucatán (PY) es un área endémica de leishmaniasis cutánea localizada, conocida localmente como "úlcera de los chicleros" (Biagi et al. 1965), en Quintana Roo la Secretaría de Salud Estatal cada año reporta un gran número de casos en sus diferentes localidades. Las personas infectadas son principalmente campesinos que realizan sus actividades en la selva. A pesar de lo mencionado anteriormente en Quintana Roo sólo se tiene información de la presencia de los flebotominos en algunas localidades (Biagi et al. 1965; Cruz et al. 1994; Ibáñez-Bernal 2000; SánchezGarcía et al. 2010), razón por la cual se plantearon los objetivos de conocer la fauna de flebotominos de Quintana Roo, determinando la riqueza y la abundancia en las tres zonas en que se dividió el estado.

\section{MATERIAL Y MÉTODOS}

Área de estudio. El estado de Quintana Roo cubre la parte oriental de la PY, se ubica entre $18^{\circ}$ y $22^{\circ}$ latitud norte y $87^{\circ}$ y $89^{\circ}$ longitud oeste. Tiene un clima cálido subhúmedo con un promedio anual de temperatura de $22^{\circ} \mathrm{C}$, y una precipitación de 450 $\mathrm{mm}$ a $1500 \mathrm{~mm}$ al año. La vegetación de la región sur de Quintana Roo es principalmente selva alta subperennifolia, en el resto del estado la selva mediana subperenni- 
folia abarca la mayor parte, aunque también se presentan parches de selvas medianas subcaducifolias. Cada uno de estos tipos de vegetación se distribuye a lo largo del gradiente de precipitación en el estado (Carnevali et al. 2003).

Los muestreos en campo se realizaron en los meses de enero a abril del 2008, los cuales corresponde al final de la época de nortes e inicio de la época de secas. Se visitaron 18 localidades del estado de Quintana Roo, el cuál se dividió para los análisis en tres zonas con seis localidades cada una; zona norte: 1 Solferino, 2 Central Vallarta, 3 Cobá, 4 Macario Gómez, 5 Francisco I. Madero, 6 Saban; zona centro: 7 Chumpon, 8 Puerto Arturo, 9 Chunhuhub, 10 San Antonio Nuevo, 11 San Isidro Poniente, 12 Petcacab; zona sur: 13 Cinco de Mayo, 14 Altos de Sevilla, 15 San Pedro Peralta, 16 Laguna Guerrero, 17 Caobas, 18 Tres Garantías.

En cada localidad se recolectó en dos sitios durante tres noches continuas, en el horario de 18:00 a 22:00 h, utilizando cuatro trampas Disney (Disney 1966), una Shannon (Shannon 1939), dentro estuvieron dos personas recolectando las muestras y cuatro trampas de luz CDC (Sudia \& Chamberlain 1962) en cada sitio respectivamente. Las trampas CDC y Disney, estuvieron acomodadas en un transecto a cada $20 \mathrm{~m}$ intercalando una trampa con otra. Todo el material que se recolectó se sacrificó con los vapores de éter y una vez seleccionado el material, se conservó en alcohol etílico al 70\%. Una vez en el laboratorio la totalidad del material se montó para su identificación usando las técnicas curatoriales convencionales (Ibáñez-Bernal 1999), y se identificaron utilizando claves taxonómicas (Young \& Duncan 1994; IbáñezBernal 2000, 2005a y b). El material está depositado en dos colecciones: la colección de artrópodos del Museo de Zoología del Colegio de la Frontera Sur unidad Chetumal con número de registro QNR.IN.017.0497 y en la Universidad Autónoma de Nuevo León.

Para la estimación de la riqueza de especies se utilizó una curva de acumulación de especies (Colwell 2004). La acumulación de especies es una herramienta utilizada para describir el comportamiento asintótico de los valores de la riqueza de especies en una localidad, de acuerdo con la medida en que el esfuerzo de muestreo se acumula. Las especies esperadas se calcularon con la fórmula de Clench (1979).

Las curvas de dominancia $\mathrm{K}$ se usaron para observar la dominancia de especies entre las localidades, y por medio de la forma que tenga la curva (exponencial o sigmoide) se puede distinguir la diversidad de las localidades. Los análisis se realizaron con el programa PRIMER versión 6.1.6 (Plymouth Routines in Multivariate Ecological Research, Clarke \& Gorley 2001).

\section{RESULTADOS}

Los muestreos se realizaron durante un total de 54 noches, en los cuales se colectaron 14,532 individuos pertenecientes a 20 especies comprendidas en dos géne- 
ros Brumptomyia y Lutzomyia, de la subfamilia Phlebotominae. Cuatro especies son registradas por primera vez para Quintana Roo: Lutzomyia beltrani (Vargas y Díaz-Nájera 1955), Lutzomyia serrana (Damasceno \& Arouck 1949), Lutzomyia itza Ibáñez-Bernal, May-Uc y Rebollar-Téllez 2010 y Lutzomyia maya IbáñezBernal, May-Uc y Rebollar-Téllez 2010, siendo éstas dos últimas especies nuevas (Ibáñez-Bernal et al. 2010). En la trampa Shannon se capturó el 87\% de los ejemplares, en la trampa de luz CDC el $12 \%$ y en la trampa Disney el $1 \%$. El total de hembras capturadas suma el $68 \%$, manteniendo la mayor abundancia en las diferentes trampas, Shannon el $68.5 \%$, CDC el $63 \%$ y Disney el $92 \%$ respectivamente.

Tres especies contribuyeron con más del $81 \%$ a la abundancia total; Lutzomyia shannoni (Dyar 1929), Lutzomyia ovallesi (Ortiz 1952) y Lutzomyia cruciata (Coqui1lett 1907). Las especies más comunes presentes en todas las localidades muestreadas fueron L. shannoni, L. cruciata y L. deleoni (Fairchild \& Hertig 1947). Otras especies como Lutzomyia steatopyga (Fairchild \& Hertig 1958) y Brumptomyia mesai Sherlock 1962, se registraron en 17 y 15 localidades, respectivamente.

En la zona norte se encontraron 19 de las 20 especies registradas, y dos de estas especies son exclusivas (Lutzomyia beltrani y Lutzomyia itza); esta zona comparte dos especies con la zona centro (L. serrana y Lutzomyia trinidadensis (Newstead 1922) y una especie con la zona sur (Lutzomyia panamensis (Shannon 1926)). En las zonas centro y sur se capturaron 16 especies en cada una. La zona centro no tiene especies exclusivas, mientras que la zona sur presentó a Lutzomyia permira (Fairchild \& Hertig1956) como especie exclusiva (Fig. 1).

Las localidades con mayor número de especies fueron: Cobá en la zona norte, Chumpon en la zona centro y San Pedro Peralta en la zona sur. Las localidades donde se registró el menor número de especies fueron: Saban en la zona norte, Puerto Arturo en la zona centro y Caobas en la zona sur. En la zona norte dos localidades presentan especies que no están en otras localidades de la misma zona (Solferino, L. panamensis y Cobá, L. serrana). Chumpon tiene dos especies que no comparte con las localidades del centro ( $L$. ovallesi y L. serrana) y en Chunhuhub se halló una especie que no está presente en las otras cinco localidades del centro del estado (Brumptomyia hamata (Fairchild \& Hertig 1947)). San Pedro Peralta presentó a Lutzomyia cratifer (Fairchild \& Hertig 1961) y B. hamata como exclusivas en las localidades de la zona sur (Fig. 2, Cuadro 1).

Las especies más abundantes por zona, en orden descendente, fueron: Zona sur $L$. ovallesi, L. shannoni, L. cruciata y L. olmeca olmeca (Vargas y Díaz-Nájera 1959). Zona centro $L$. shannoni, B. mesai y L. cratifer fueron las más abundantes. Zona norte L. shannoni, L. cruciata y B. mesai. Se observó que Lutzomyia longipalpis (Lutz y Neiva 1912) se colectó en ocho localidades, ésta tiene un gran número de individuos en Cobá en la zona norte. Lutzomyia deleoni se presentó en todas las localidades aun- 


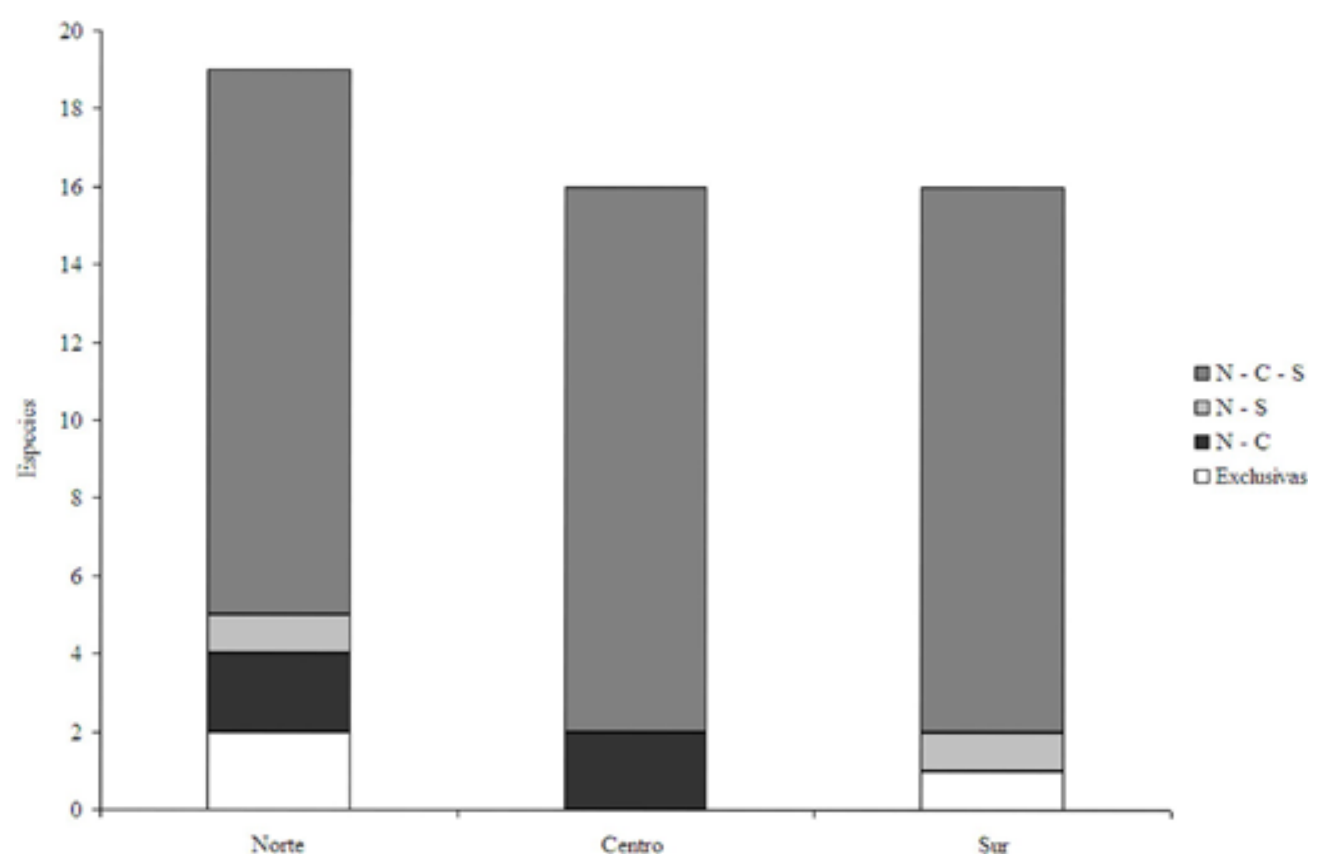

Figura 1. Especies exclusivas por zona y número de especies por zona, N-C son las especies compartidas entre la zona norte y la centro, N-S especies compartidas entre la zona norte y sur, N-C-S especies que se encuentran en las tres zonas.

que su representación fue en promedios bajos y constantes. Lutzomyia steatopyga se distribuyó por todo el estado y los promedios altos se encontraron en las zonas norte y centro (Cuadro 1).

Las mayores abundancias promedio se registraron en Macario Gómez (zona norte), y San Pedro Peralta (zona sur), la localidad de Saban fue la que registró menor promedio de abundancia. Las localidades de la zona sur presentaron mayor variabilidad en las abundancias por encima del centro y el norte. Las zonas no manifiestan ninguna tendencia clara que muestre un patrón por abundancia. Sin embargo, la zona norte presentó la mayor abundancia total y la zona centro con la abundancia más baja (Fig. 3).

El mayor promedio de especies se observó en San Pedro Peralta de la zona sur y Macario Gómez en la zona norte. El menor promedio de la zona sur se obtuvo en Laguna Guerrero, en la zona centro Puerto Arturo y San Isidro Poniente y en la zona norte Saban. En general existe mucha variabilidad entre localidades dentro de las zonas y entre zonas a lo largo del estado (Fig. 4). 

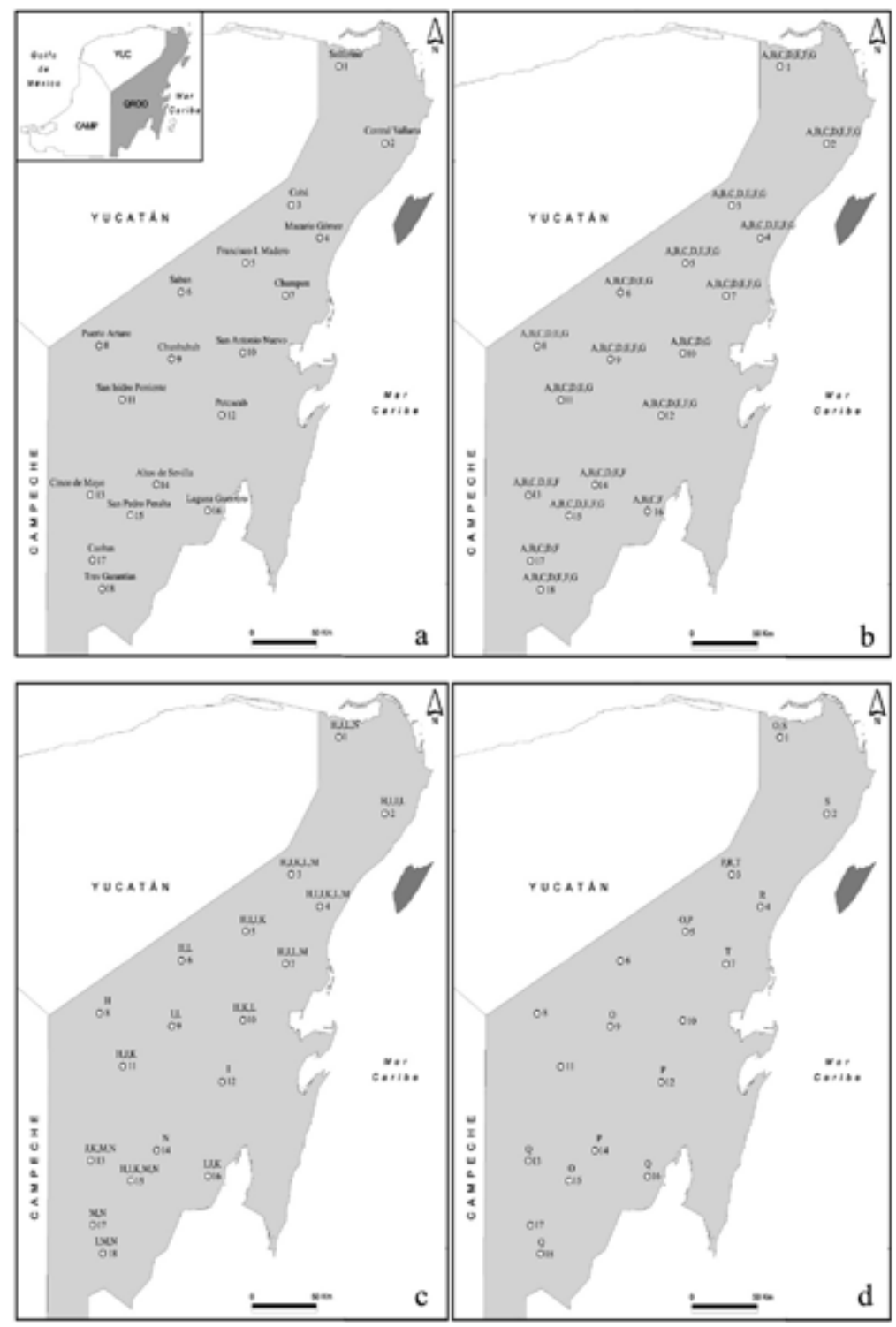

Figura 2. Mapas de distribución de localidades muestreadas y especies en Quintana Roo: a) mapa en el que se muestran enumeradas las 18 localidades del estado de Quintana Roo en las que se realizaron los muestreos. b) distribución de Lutzomyia shannoni (A), L. cruciata (B), L. deleoni (C), L. steatopyga (D), Brumptomyia mesai (E), L. olmeca olmeca (F) y L. undulada (G). c) distribución de L. cratifer $(\mathrm{H})$, L. carpenteri $(\mathrm{I})$, L. cayennensis maciasi $(\mathrm{J})$, L. longipalpis $(\mathrm{K})$, L. trinidadensis $(\mathrm{L})$, L. ovallesi $(\mathrm{M})$ y L. panamensis $(\mathrm{N})$. d) distribución de B. hamata $(\mathrm{O})$, L. maya $(\mathrm{P}), L$. permira $(\mathrm{Q}), L$. beltrani $(\mathrm{R})$, L. itza $(\mathrm{S})$ y L. serrana $(\mathrm{T})$. 


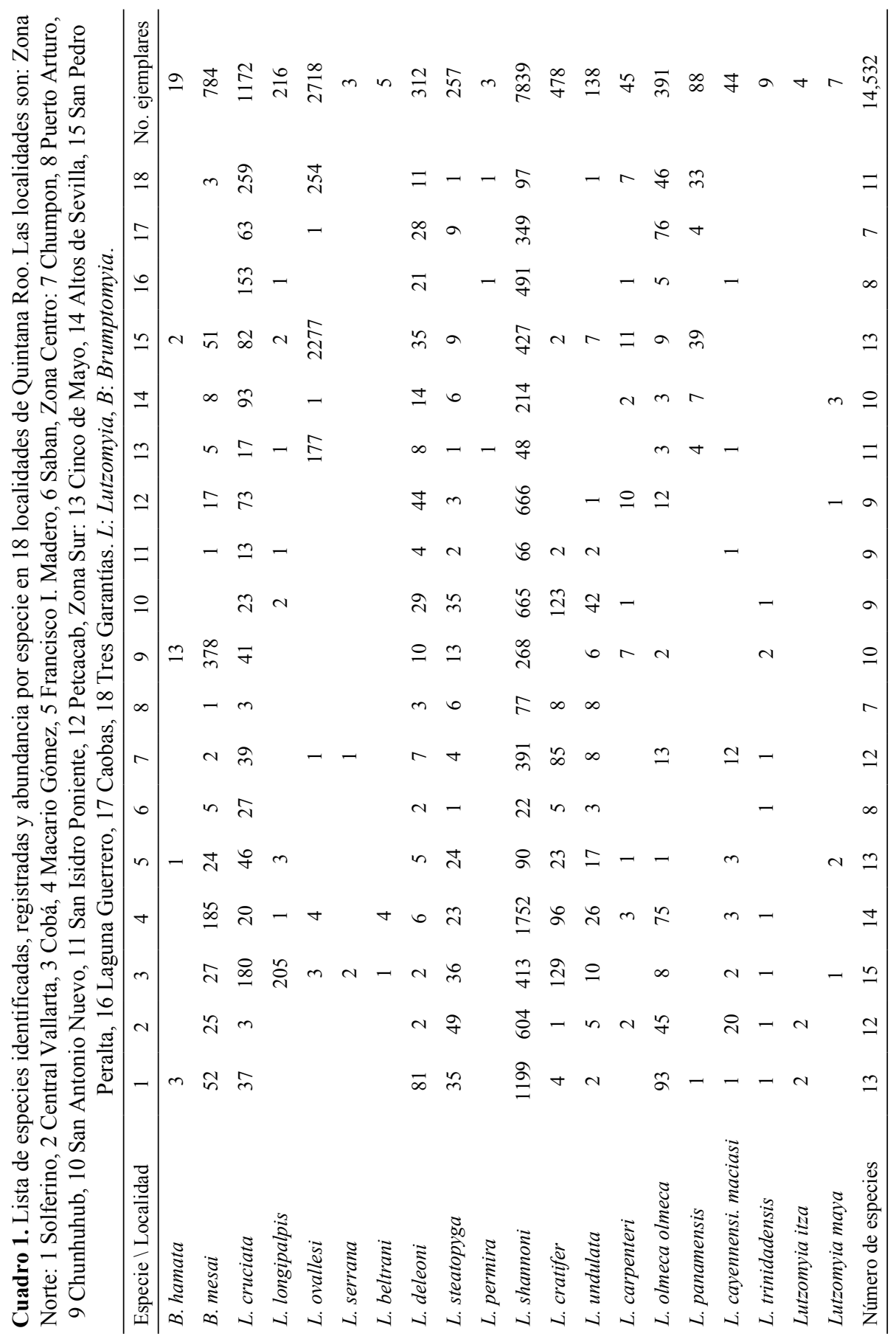




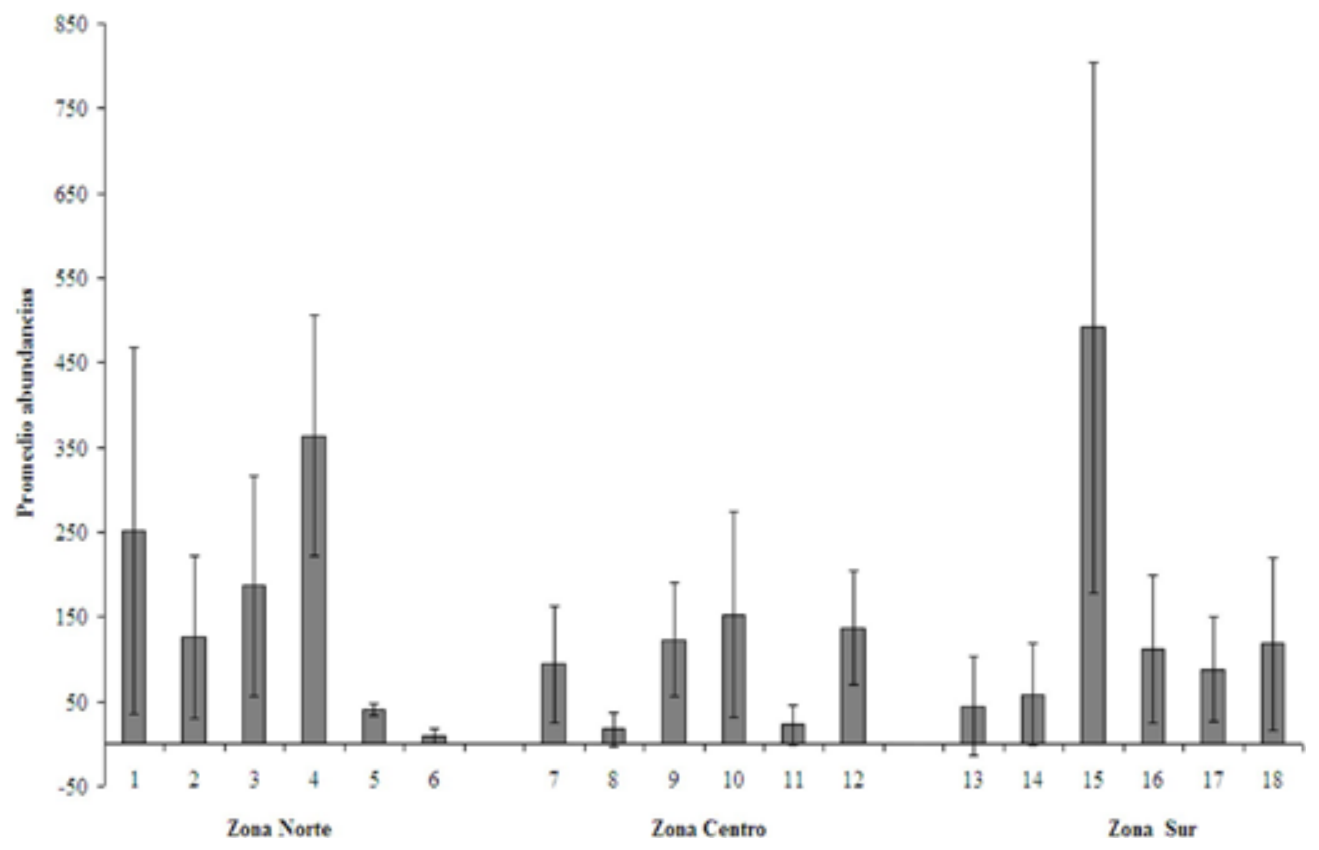

Figura 3. Patrón de variabilidad espacial de la abundancia promedio a escala de localidad. Las líneas de las barras indican las unidades de desviación estándar.

El patrón de dominancia por zona no está definido. En la zona centro en seis localidades, una sola especie dominó la abundancia con más del 50\% (L. shannoni) y en Chunhuhub dominó B. mesai (Fig. 5), en siete localidades L. shannoni tiene más del $70 \%$ de la abundancia y en una localidad $L$. ovallesi alcanza más del $70 \%$ de abundancia. En cinco localidades entre el $60 \%$ y $70 \%$ de la abundancia pertenece a una especie, cuatro localidades dominada por L. shannoni y una por L. ovallesi. Cuatro localidades entre el $30 \%$ y $40 \%$ de la abundancia lo presenta una especie, en tres localidades de la zona norte fue $L$. shannoni y en la sur $L$. cruciata.

En la curva de acumulación de especies se observa que la asíntota se ha alcanzado después de 108 muestras y veinte especies encontradas en los diferentes sitios de colecta, utilizando la fórmula de Clench (1979), se calculó que 20.7 fueron las especies esperadas en el área de estudio. Comparando el número de especies capturadas con el número de especies esperadas se obtuvo más del 96.6\% de las especies. Por lo tanto, el número de especies y el número de muestras tomadas confiere un fuerte soporte de la confiabilidad del estudio que se realizó y es un porcentaje muy representativo para el área (Fig. 6). 


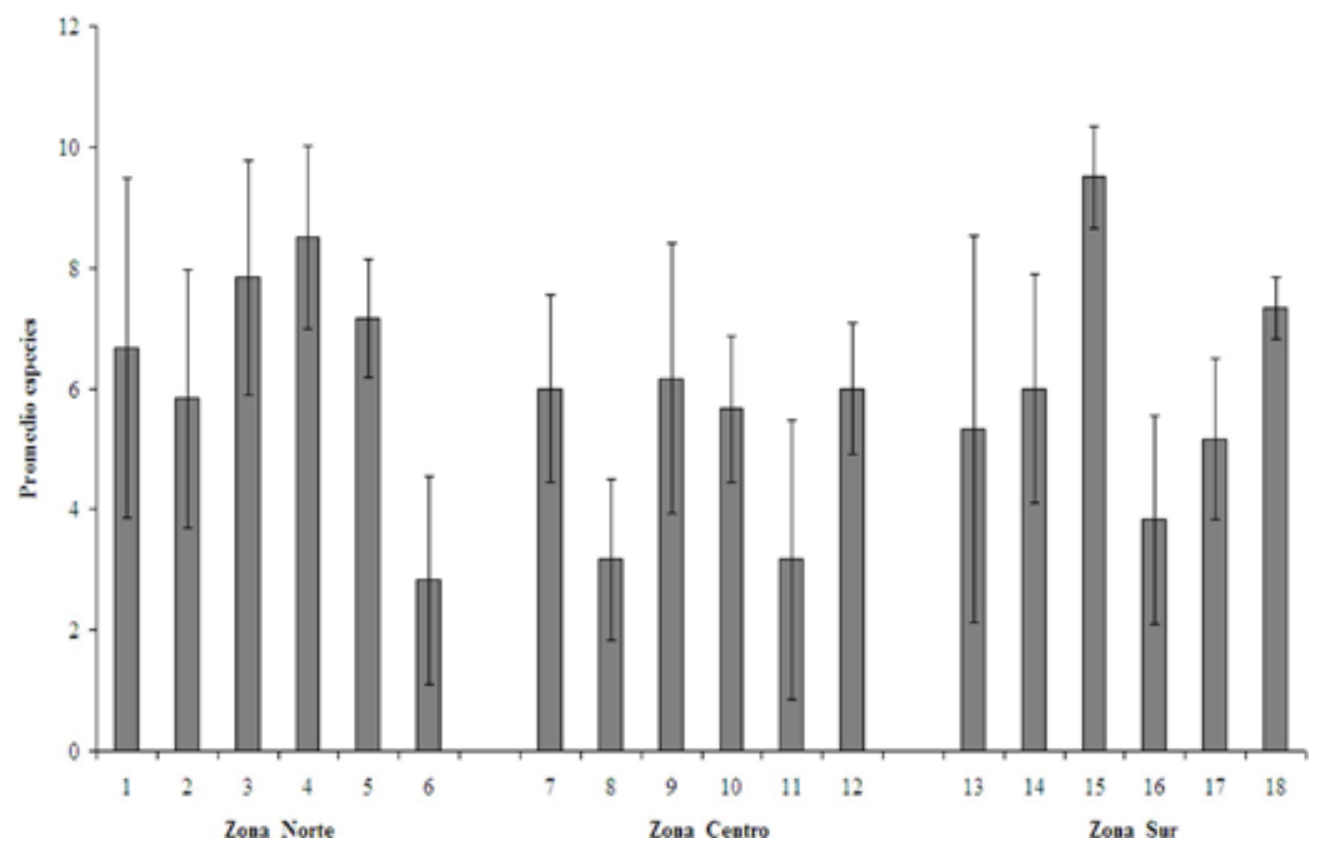

Figura 4. Patrón de variabilidad espacial en el número de especies promedio a escala de localidad. Las líneas de las barras indican las unidades de desviación estándar.

\section{DISCUSIÓN}

En trabajos previos se habían registrado 19 especies de flebotominos pertenecientes a los géneros Brumptomyia y Lutzomyia (Cruz-Ruiz et al. 1994; Ibáñez-Bernal 1999, 2000, 2002, 2003; Sánchez-García et al. 2010). Los resultados de este estudio permitieron incrementar a 23 el número de especies registradas para Quintana Roo con cuatro nuevos hallazgos. Para la PY L. beltrani es nuevo registro. Actualmente las especies de flebotominos para la PY son 32, de éstos, suman 23 especies las informadas para Quintana Roo con los nuevos registros aportados en este estudio, 16 para Campeche y 11 para Yucatán. En Belice se han registrado 25 especies y ocho de ellas diferentes a las presentes en Quintana Roo (Ibáñez-Bernal 2001b). Del total de las especies en la Península de Yucatán, Quintana Roo tiene el 72\% y en relación con todo México, Quintana Roo tiene un 55\% de las especies (Cruz-Ruiz et al. 1994; Ibáñez-Bernal 1999, 2000, 2001a, 2001b, 2002, 2003; Ibáñez-Bernal et al. 2004; Sánchez-García et al. 2010; Rebollar-Téllez et al. 1996, 2004, 2005, 2006; Pech-May et al. 2010; Peraza 2008).

El número de especies capturadas fue muy similar entre las trampas Shannon y $\mathrm{CDC}$, dos especies encontradas en la trampa de luz no se capturaron con la trampa 


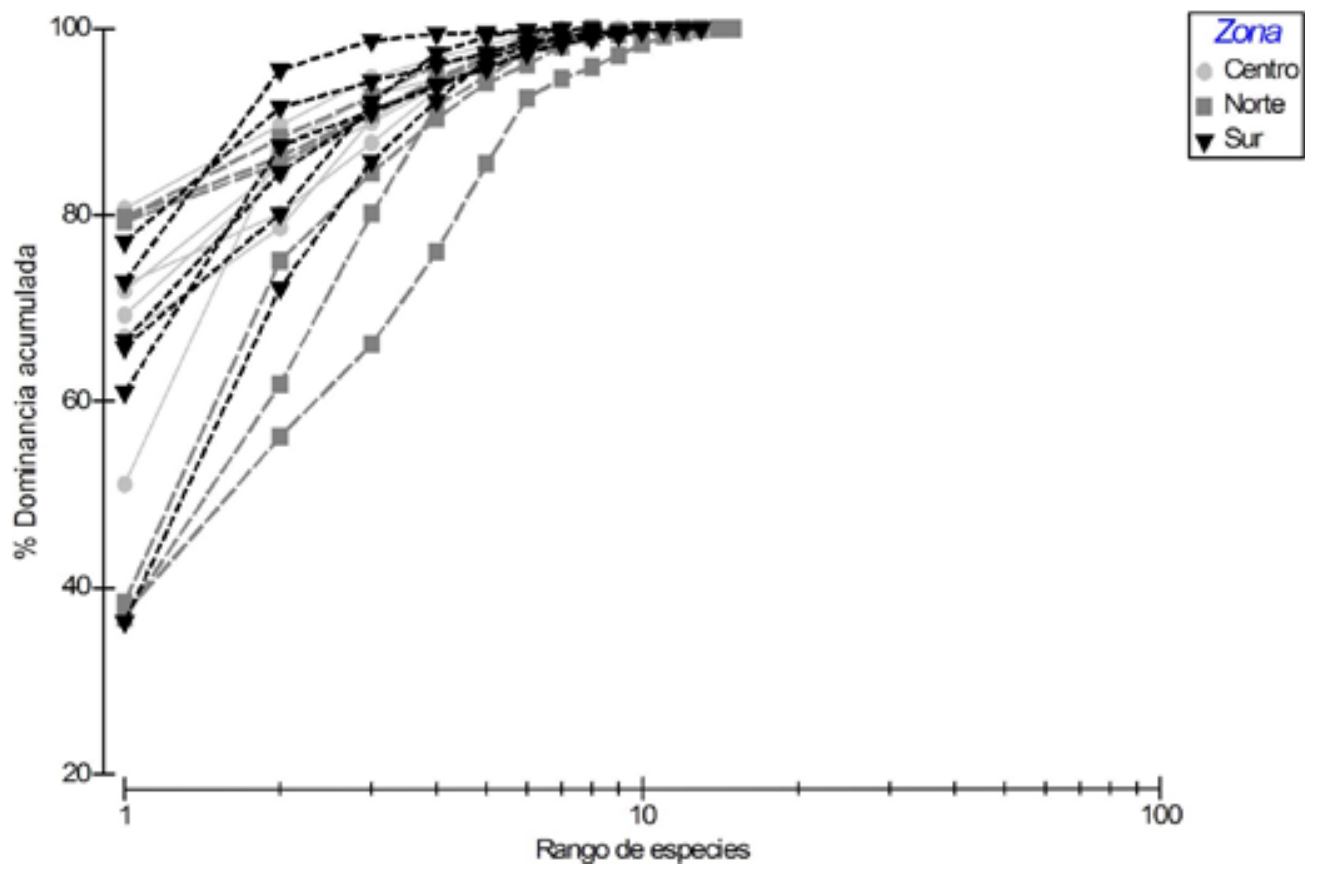

Figura 5. Curvas de dominancia K de las 18 localidades de Quintana Roo, separadas por zonas.

Shannon (L. beltrani y L. itza), mientras que en las trampas CDC no se capturo una especie recolectada de la Shannon (L. serrana). La proporción de sexos fue similar también. En la trampa Disney se capturaron ocho especies, siendo L. olmeca olmeca la más abundante, en otros estudios realizados en la PY en la trampa Disney solo se ha capturado esta especie (Rebollar-Téllez et al. 2005; Sánchez-García et al. 2010).

Las especies más abundantes para Quintana Roo en este estudio fueron L. shannoni $53 \%$, L. ovallesi $19 \%$ y L. cruciata con el $8.7 \%$. Dentro de cada zona las proporciones y las especies variaron, en la zonas norte y centro $L$. shannoni fue la más abundante. En la zona sur L. ovallesi concentrado en una localidad (San Pedro Peralta) que contribuyó con $85 \%$ de la abundancia. Esta proporción en esta localidad probablemente se deba a factores como la anidación (Pavlosky 1959), esta hipótesis explica que "donde los factores bióticos y abióticos se conjugan en un área se favorecen las altas densidades de una especie". Otras especies abundantes fueron B. mesai, que en la zona centro y norte ocuparon el segundo y tercer lugar respectivamente, y L. cratifer tercer y cuarto más abundante en las zonas centro y norte, respectivamente. Comparando este trabajo con otros que se realizados en la PY se observan marcadas diferencias en área estudiada, diseño de muestreo y tamaño de muestra, por lo que no 


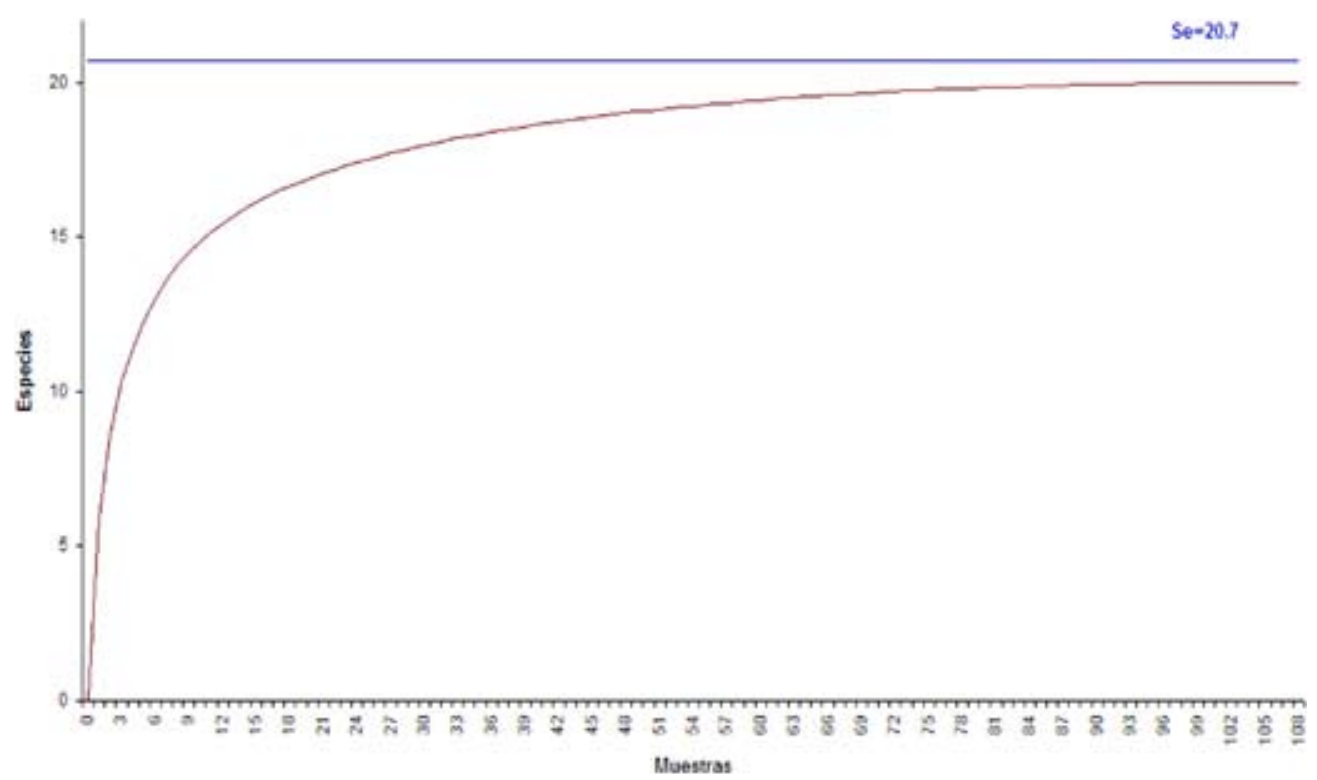

Figura 6. Curva de acumulación de especies con las especies esperadas (Se) calculada con la formula de Clench.

sería válido hacer comparaciones directas ya que el patrón de dominancia es fuertemente influenciado por estos tres aspectos metodológicos. No obstante, es importante hacer notar algunas consideraciones, todos estos estudios presentan al mismo grupo de especies con diferentes proporciones de abundancias y la mayoría son incriminados ó sospechosos de ser vectores potenciales de leishmaniasis en México y Centro América. Estos trabajos son los de Cruz-Ruiz et al. (1994), observando a L cruciata con $47 \%$ y L. shannoni $44 \%$; Rebollar-Téllez et al. (2005) obtuvieron a L. panamensis $30 \%$, L. cruciata $18 \%$, L. olmeca olmeca $11 \%$ y L. ovallesi $9 \%$; Sánchez-García et al. (2010) colectaron a L. olmeca olmeca, $57 \%$, L. shannoni $15 \%$ y L. cruciata $14 \%$; Pech-May et al. (2010) capturaron a L. panamensis 30\%, L. olmeca olmeca $28 \%, L$. shannoni y L. cruciata $15 \%$ cada uno; Peraza (2008) registró a L. shannoni $48 \%, L$. cruciata $29 \%$, L. olmeca olmeca $16 \%$ y L. panamensis $7.5 \%$. También resalta que estas especies numéricamente dominantes tienen una amplia distribución en la PY y que el efecto temporal, $i$. e. de una época a otra no afecta la composición del grupo dominante sino únicamente su abundancia (Sánchez-García et al. 2010). B. mesai y L. cratifer solo aparecen como numéricamente importantes en la zona centro y norte de Quintana Roo, B. mesai se desarrollan en madrigueras de armadillos y son atraídos por trampas luminosas (Young \& Duncan 1994), por lo que probablemente las tram- 
pas CDC fueron colgadas cerca de madrigueras de estos animales en las localidades donde se obtuvieron mayor abundancia. Se desconoce la biología y ecología de $L$. cratifer, pero en este estudio se capturó la mayor cantidad en la trampa Shannon. En la zona sur de Quintana Roo L. ovallesi es numéricamente dominante, aunque de distribución restringida, ya que en una localidad se capturó más del $80 \%$ de los ejemplares, la mayor cantidad se capturó en trampa Shannon, a diferencia de Sánchez-García et al. (2010), quienes encontraron mayor número de L. ovallesi atraída por la luz. En otros estudios en la PY se conoce por ser una especie antropófila y sospechosas de ser vector de leishmaniasis (Pech-May et al. 2010; Rebollar-Téllez et al. 2005).

La presencia de especies de flebotominos a lo largo de un área se debe a varios factores; como la vegetación, la presencia de mamíferos de los que se alimentan, a las variaciones del clima, la cantidad de materia orgánica en el suelo y la topografía (Azevedo et al. 2002; De Luca et al. 2003; Feliciangeli 2004; Ivovic et al. 2007; Valderrama et al. 2008; Travi et al. 2002).

Los nuevos registros para el estado, como L. beltrani, Lutzomyia itza y L. serrana en la zona norte, podrían explicarse por sus requerimientos específicos del hábitat. Por ejemplo, $L$. beltrani solo se colectó en dos localidades donde existen gran cantidad de oquedades formados en el suelo como se muestran en la figura 7 (observación personal), esta especie se ha colectado en cuevas en otras regiones del país y Belice (Ibáñez-Bernal, 2000; Williams 1979). Lutzomyia itza es una nueva especie colectada en bajos números únicamente en dos localidades (Solferino y Central Vallarta) y se desconoce su biología y ecología.

Otras especies también se observaron con distribuciones restringidas y números reducidos, aunque se han registrado en trabajos previos. Como es el caso de $L$. serrana, L. trinidadensis presentes en las zonas norte y centro, aunque la segunda ha sido anteriormente registrada en áreas periurbanas de la zona sur (Sánchez-García et al. 2010). L. panamensis se presenta en una localidad de la zona norte y en la mayoría de las del sur. Sin embargo, en otros trabajos ha sido registrada en el centro de Quintana Roo (Cruz-Ruiz et al. 1994; Ibáñez-Bernal 2000).La zona sur presenta a $L$. permira como exclusiva, recolectada en tres localidades y registrada anteriormente para Chetumal, siendo una de las especies menos abundantes en varios estudios en la PY (Rebollar-Téllez et al. 2004; Rebollar-Téllez et al. 2005; Pech-May et al. 2010; Sánchez-García et al. 2010). Estas especies son raras, con distribución restringida y numéricamente contribuyen poco a los patrones de dominancia. Los factores que controlan su distribución pueden ser desde ambientales hasta interacciones biológicas, o tal vez no se recolectaron por que las metodologías utilizadas no fueron las apropiadas para su captura, pero no hay elementos que permitan establecerlos.

Antes de realizarse este trabajo se tenía la idea de que la mayor abundancia y riqueza se encontraría en la zona sur del estado debido a las condiciones que prevalecen en la zona (vegetación de selva alta y mayor porcentaje de precipitación), sin 


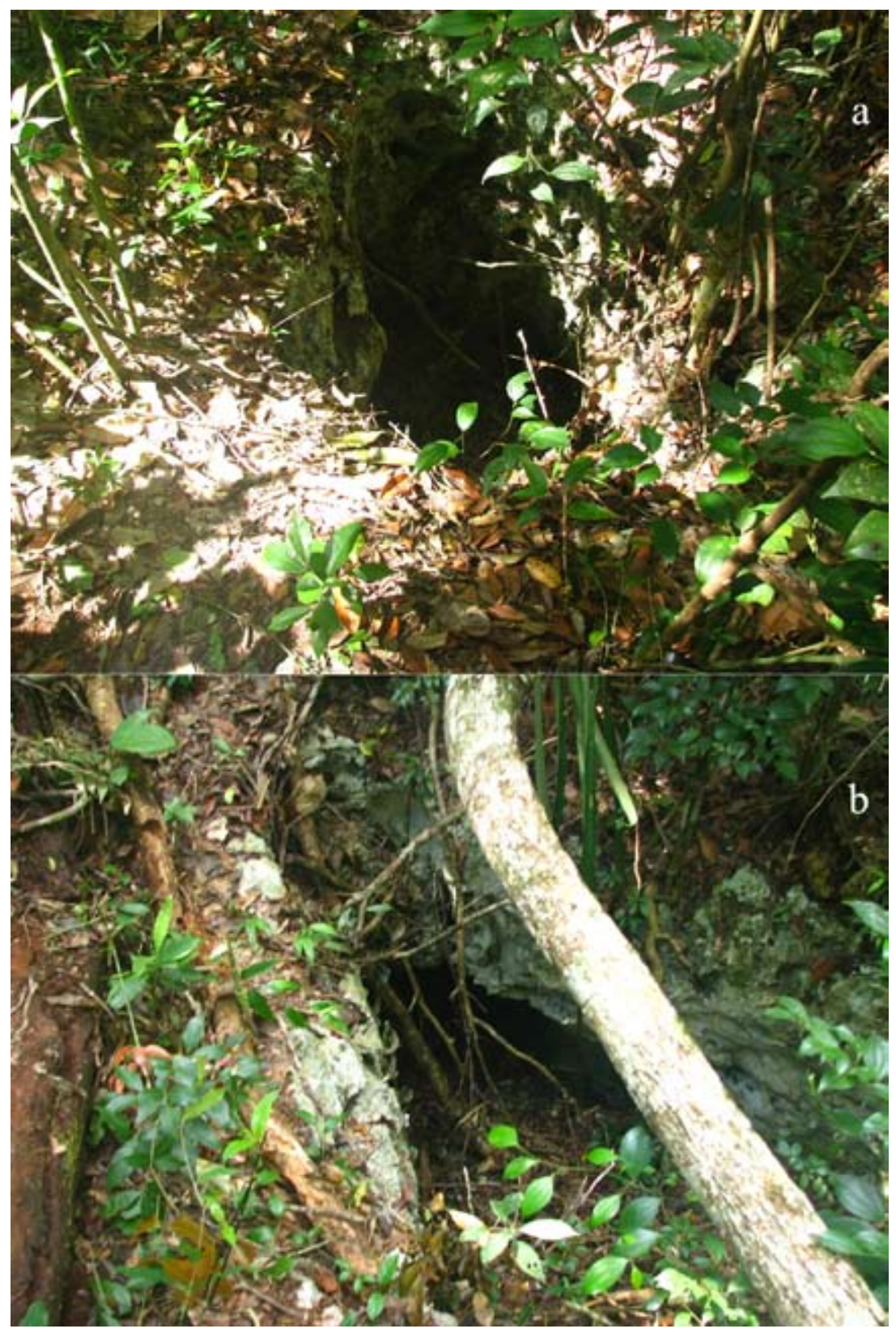

Figura 7. Oquedades naturales en el suelo (a y b) que se presentan en algunas localidades de la Zona norte de Quintana Roo, en ocasiones formando pequeñas cuevas (b) donde se acumula gran cantidad de materia orgánica, formando microambientes que favorecen a los flebotominos. 
embargo la mayor abundancia y riqueza fue registrada en la zona norte del estado, la zona centro y sur registraron igual número de especies y la zona centro la menor abundancia. Estas diferencias con lo esperado probablemente se deben a dos causas: 1) La disminución de la riqueza y abundancia esperada en el sur podría deberse a la perturbación provocada por el huracán Dean en agosto de 2007, ya que nuestras colectas se hicieron cinco meses después y cuando aún era observable el impacto del disturbio sobre la vegetación. En estudios realizados por Barberena-Arias \& Aide (2002) observaron que la riqueza y la abundancia de insectos después de un huracán disminuyen y vuelve a las proporciones anteriores hasta después de un año del disturbio. 2) Los muestreos se extendieron hasta mediados de abril, por lo cual varias localidades del centro fueron muestreados al comienzo de la temporada de secas, dónde se obtuvieron menor número de ejemplares, propiciando la menor abundancia en la zona centro. En diversos estudios se ha observado una estacionalidad en las abundancias de los flebotominos (McHugh et al. 2001; Salomón et al. 2004; Sánchez-García et al. 2010).

El número de especies que se obtuvo en este estudio fue representativo para el estado, observando la curva de acumulación de especies se distingue que la línea ha alcanzado la asíntota por lo que la aparición de nuevas especies podría ser mínima aun si se hubiese aumentado el número de muestreos. En trabajos previos realizados en la PY indican que las mayores abundancias se presentan durante los meses de noviembre a febrero (Rebollar-Téllez et al. 1996; 2005; Sánchez-García et al. 2010)

Al menos siete especies de flebotominos se distribuyen a lo largo del estado, dentro de ellas están las especies más abundantes, que en algunos casos fueron dominantes en una sola zona. Otras tuvieron pocos ejemplares pero se distribuyeron en todas las zonas del estado. Algunas especies se consideran raras por su poca abundancia y distribución restringida a unas localidades dentro de una zona. Dentro de las especies de amplia distribución y gran abundancia están las especies que son incriminadas o sospechosas como vectores de leishmaniasis tanto en la PY como en otras regiones de América. La abundancia y número de especies que se encontraron en la zona sur probablemente se vió afectada por el paso de huracán Dean meses atrás. Lo que probablemente propició la disminución de abundancias y número de especies en esta zona.

AGRADECIMIENTOS. Esta investigación recibió apoyo financiero de los proyectos: "Determinación de Zonas de Riesgo de Transmisión de Leishmaniasis Mediante el Análisis de la Distribución Geográfica de los Insectos Vectores y su Capacidad Vectorial”. Fondo Mixto Gobierno del Estado de Quintana Roo y Conacyt (QROO-2005-C01-19166), así como de "Distribución geográfica y biodiversidad de insectos (Diptera: Psychodidae) vectores de leishmaniasis en el estado de Quintana Roo". PAYCIT-UANL (CN023-09) en ambos proyectos: Responsable Dr. Eduardo A. Rebollar-Téllez. Al Fomix CONACYT Quintana Roo por la beca nivel II otorgada al primero de los autores. Se agradecen los valiosos comentarios de Dr. Sergio Ibáñez-Bernal y del Dr. Julio Rojas. Se hace un agradecimiento por la hospitalidad 
y ayuda de los ejidatarios y comisarios de las localidades, así como a los biólogos David Moo Llanes, Ana Celia Montes de Oca, René Cortéz León y a Irving May Concha, por su ayuda en las colectas de campo. Al Posgrado de ECOSUR. Al CONACYT por la beca otorgada durante los estudios de maestría. Al museo de Zoología de ECOSUR Chetumal por las facilidades otorgadas durante la preparación de los ejemplares.

\section{LITERATURA CITADA}

Azevedo, A. C. R., N. A. Souza, C. R. V. Meneses, W. A. Costa, S.M. Costa, J. B. Lima \& E. F. Rangel. 2002. Ecology of sand flies (Diptera: Psychodidae: Phlebotominae) in the north of the State of MatoGrosso, Brazil. Memórias do Instituto Oswaldo Cruz, 97: 459-464.

Barberena-Arias, M. F. \& T. M. Aide. 2002. Variation in species and trophic composition of insect communities in Puerto Rico. Biotropica, 34: 357-367.

Biagi F. F., A. M. De Biagi \& F. Beltrán. 1965. Phlebotomus flaviscutellatus transmisor natural de Leishmania mexicana. Prensa Médica Mexicana, 30: 267-272.

Carnevali, G., I. M. Ramírez \& J. A. Gonzáles-Iturbe. 2003. Flora y vegetación de la Península de Yucatán, pp. 53-68. In: M. Colunga-García \& A. Larqué Saavedra (Eds.). Naturaleza y sociedad en el área Maya, Pasado, presente y futuro. Academia Mexicana de Ciencias.México.

Clarke K. R. \& R. N. Gorley. 2001 PRIMER v 6.1.6: User manual/ tutorial. PRIMER-E, Plymouth.

Clench, H. K. 1979. How to make regional list of butterflies: some thoughts. Journal of Lepidoptera Society, 33: 215-231.

Colwell, R. K. 2004. EstimateS (Version 8.00). http://viceroy.eeb.uconn.edu/estimate

Cruz-Ruiz, A. L., J. E García-Rejón, P. Manrique-Saide \& J. Pérez-Mutul, 1994. Taxonomical identification of anthropophilic species of Lutzomyia in Quintana Roo, Peninsula of Yucatán, México. Revista Biomédica, 5: 127-131.

De Luca, A. S., H. L. Vasconcelos \& T. V. Barrett. 2003. Distribution of sandflies (Diptera: Psychodidae) in forest remnants and adjacent matrix habitats in Brazilian Amazonia. Brazil Journal Biology, 63: 401-410.

Disney, R. H. L. 1966. A trap for phlebotominae sandflies attracted to rats. Bulletin of EntomologicalResearch, 56: 445-451.

Duran, R. \& I. Olmsted. 1999. Vegetación de la Península de Yucatán, pp. 187-194. In: A. García de Fuentes, J. Córdoba y Ordóñez, P. Chico Ponce de León (Eds.). Atlas de procesos territoriales de Yucatán. Universidad Autónoma de Yucatán. Mérida, Yucatán, México.

Feliciangeli, M. D. 2004. Natural breeding places of Phlebotominae sandflies. Medical Veterinary Entomology, 18: 71-80.

Gibson, G. \& J. Torr. 1999. Visual and olfactory responses of haematophagousdiptera to host stimuli. Medical VeterinaryEntomology, 13: 2-23.

Ibáñez-Bernal, S. 1999. Phlebotominae (Diptera: Psychodidae) de México. I.-Brumptomyia Franca y Parrot; Lutzomyia Franca, las especies de L. (Lutzomyia) Franca y del grupo verrucarum. Folia Entomológica Mexicana, 107: 61-116.

Ibáñez-Bernal, S. 2000. Los Phlebotominae (Diptera: Psychodidae) de México. Tesis Doctoral Universidad Nacional Autónoma de México. 288 pp.

Ibáñez-Bernal, S. 2002. Phlebotominae (Diptera: Psychodidae) de México. III.- Las especies de Lutzomyia (Psathyromyia) Barreto, del grupo del Aragoi, de L. (Trichopygomyia) Barreto, del grupo Dreisbachiy de L (Nyssomyia) Barreto. Folia Entomológica Mexicana, 41: 149-183.

Ibáñez-Bernal, S. 2003. Phlebotominae (Diptera: Psychodidae) de México. IV.- Las especies de Lutzomyia (Psychodopygus) Mangabeira, L. (Micropygomyia) Barreto, Lutzomyia grupo Oswaldoi, 
May-Uc et al.: Distribución de flebotominos en Quintana Roo, México

L. (Helcocyrtomyia) Barreto y especies del género sin agrupar. Folia Entomológica Mexicana, 42: 109-152.

Ibáñez-Bernal, S., G. Rodríguez-Domínguez, C. H. Gómez-Hernández \& J. R. Ricardez-Esquinca. 2004. First record of Lutzomyia evansi (Nuñez-Tovar 1924) in Mexico (Diptera: Psychodidae, Phlebotoninae). Memórias do Instituto Oswaldo Cruz, 99: 127-129.

Ibáñez-Bernal, S. 2005a. Phlebotominae (Diptera: Psychodidae) de México. V.- Clave ilustrada para la identificación de los machos de Lutzomyia Franca. Folia Entomológica Mexicana, 44: 49-66.

Ibáñez-Bernal, S. 2005b. Phlebotominae (Diptera: Psychodidae) de México. VI.- Clave ilustrada para la identificación de las hembras de Lutzomyia Franca. Folia Entomológica Mexicana, 44: 195-212.

Ibáñez-Bernal, S., E. May-Uc \& E. A. Rebollar-Téllez. 2010. Two new species of phlebotominae sand flies (Diptera: Psychodidae, Phlebotominae) from Quintana Roo, México. Zootaxa, 2448: 26-34.

Ivovic, V., M. Patakakis, Y. Tselentis \& B. Chaniotis. 2007. Faunistic study of sandflies in Greece. Medical and Veterinary Entomology, 21: 121-124.

Organización Mundial de la Salud. 1990. Lucha contra la leishmaniasis. Organización Mundial de la Salud. Serie de informes técnicos. 793:176.

McHugh, C. P., B. F. Ontrander, R. W. Raymond \& S. F. Kerr. 2001. Population dynamics of sand flies (Diptera: Psychodidae) at two foci of leishmaniasis in Texas. Journal of Medical Entomology, 38: 268-277.

Pech-May, A., F. J. Escobedo-Ortegón, M. Berzunza-Cruz \& E. A. Rebollar-Téllez. 2010. Incrimination of four sandfly species previously unrecognized like vectors of Leishmania parasites in Mexico. Medical and Veterinary Entomology, 24: 150-161.

Peraza-Herrera, G. J. 2008. Especies de flebotominos (Diptera:Psychodidae) de importancia médica y su importancia como vectores de Leishmania mexicana (Kinetoplastida: Tripanosomatidae) en Calakmul, Campeche. Tesis de Licenciatura. Universidad Autónoma de Yucatán. Mérida, Yucatán, México. 44pp.

Rebollar-Téllez, E. A., A. Ramírez-Fraire \& F. J. Andrade-Narvaéz. 1996. A two years study on vectors of cutaneous leishmaniasis. Evidence for sylvatic transmission cycle in the state of Campeche, Mexico. Memórias do Instituto Oswaldo Cruz, 91: 555-560.

Rebollar-Téllez E. A., E. Tun-Ku, P. C. Manrique-Saide, A. Che-Mendoza, \& F. A. Dzul-Manzanilla. 2004. Further records of phlebotomid sandflies (Diptera: Phlebotomidae) from Campeche Mexico. Entomological News, 115: 283-291.

Rebollar-Téllez E. A., E. Tun-Ku, P. C. Manrique-Saide\& F. J. Andrade-Navaez. 2005. Relative abundances of sandfly species (Diptera: Phlebotominae) in two villages in the same area of Campeche, in southern Mexico. Annals of Tropical Medicine and Parasitology, 99: 193-201.

Rebollar-Téllez E. A., H. Orilla-Moguel, F. A. Dzul-Manzanilla, A. Che-Mendoza, P. ManriqueSaide \& A. Zapata-Peniche. 2006. An update on the phlebotomid sand fly (Diptera: Phlebotomidae) fauna of Yucatan, Mexico. Entomological News, 117: 21-24.

Salomón, O. D., M. L. Wilson, L. E. Munstermann \& B. L. Travi. 2004. Spatial and temporal patterns of phlebotomine sand flies (Diptera: Psychodidae) in a cutaneous leishmaniasis focus in northern Argentina. Journal of Medical Entomology, 41: 33-39.

Sánchez-García, L., M. Berzunza-Cruz, I. Becker-Fauser \& E. A. Rebollar-Téllez. 2010. Sand flies naturally infected by Leishmania (L.) mexicana in the peri-urban area of Chetumal city, Quintana Roo, México. Transactions of the Royal Society of Tropical Medicine and Hygiene, 104: 406-411.

Shannon, R. C. 1939. Methods for collecting and feeding mosquitoes in jungle yellow fever studies. American Journal of Tropical Medicine and Hygiene, 9: 131-140.

Sudia, W.D. \& R.W. Chamberlain. 1962. Battery operated light trap, an improved model. Mosquito News, 22: 126-129. 
Travi, B. L., G. H. Adler, M. Lozano, H. Cadena \& J. Montoya-Lerma. 2002. Impact of habitat degradation on Phlebotominae (Diptera: Psychodidae) of tropical dry forests in Northern Colombia. Journal of Medical Entomology, 39: 451-456

Valderrama C. A., M. Herrera \& A. Salazar. 2008. Relación entre lacomposición de especiesdel género de Lutzomyia Franca (Díptera: Psychodidae, Phlebotominae) y los diferentes tipos de vegetaciónen Panamá. Acta Zoológica Mexicana, 24: 67-78.

Williams, P. 1976. Flagellate infections in cave-dwelling sanflies (Diptera, Psychodidae) in Belize, Central America. Bulletin of Entomological Research, 65: 615-629.

Young, D. G. \& M. A. Duncan. 1994. Guide to the Identification and Geographic Distribution of Lutzomyia sand flies in Mexico, the West Indies, Central y South America (Diptera:Psychodidae). Memoirs of the American Entomological Institute, No. 54: 881pp. 